\title{
ATTITUDE ESTIMATION OF THE DELFI-n3Xt SATELLITE
}

\author{
D. Choukroun ${ }^{1,2}$, A. Maas $^{1}$, and J.M. Kuiper ${ }^{1}$ \\ ${ }^{1}$ Faculty of Aerospace Engineering \\ Delft University of Technology \\ Delft 2629 HS, The Netherlands \\ ${ }^{2}$ Mechanical Engineering Department \\ Ben-Gurion University of the Negev \\ Beer-Sheva 84105, Israel
}

\begin{abstract}
This paper presents current developments of the attitude determination algorithm for Delfi-n3Xt, TU Delft next nanosatellite. Several novel quaternion filters using Sun vector and Earth magnetic field measurements and rate gyro outputs are presented. The quaternion measurement matrix associated with each line-of-sight measurement is shown to be rank deficient. This property is exploited in order to design reduced order measurement update stages in the filters. The measurement model reduction is designed such as to preserve the statistical information. The filter covariance propagation can cope rigorously with the multiplicative process noises. The paper also describes the development of the Sun vector determination algorithm, which merges the outputs of 6 body-mounted four-quadrant Sun sensors. For each sensor, a simple algorithm allows Sun vector determination while avoiding the use of uncertain physical parameters. This algorithm takes into account geometrical imperfections linked to manufacturing limitations. A thorough error analysis of the photodiodes measurement outputs is carried out. A spacecraft Sun vector determination algorithm is proposed and illustrated, in the absence of Earth albedo effect, via Monte-Carlo simulations and experimental validation. In addition, extensive Monte-Carlo simulations illustrate the good performances of the quaternion filters using spacecraft Sun vector and Earth magnetic field measurements. The novel reduced filter shows good performances in a challenging tumbling dynamics environment, where a standard additive Kalman filter fails to converge.
\end{abstract}

\section{INTRODUCTION}

The attitude quaternion $[1$, p. 758$]$, $\mathbf{q}$, is the most popular spacecraft attitude parametrization and its mathematical modeling and filtering have been ongoing 
topics of research for more than forty years [2]. An important family of quaternion estimators has been developed using the Kalman filtering paradigm [3,4]. This approach presents known advantages such as statistically meaningful estimates, an easy estimation error covariance analysis, and the straightforward incorporation of parameters other than attitude in the estimation process, to name a few. Recently, the well-known quaternion process equation, which features state-multiplicative noises due to gyro noises in the angular velocity measurement, was reformulated in terms of an Itô stochastic differential equation [5]. This was done in order to comply with the mean-square calculus theory underlying the Kalman-Bucy filtering theory. A related result consisted of the specific Lyapunov differential equation for the quaternion second-order moment. Its driving term, which stemmed from the state-multiplicative quaternion process noise, was explicitly developed and the solution was shown to preserve a trace invariance, which express the quaternion norm invariance in the mean-square sense. Further analysis of that equation was, however, needed. Previously, another work [6] on quaternion Kalman filtering from vector observations introduced a $4 \times 4$ measurement model equation for $\mathbf{q}$, which was bilinear with respect to $\mathbf{q}$ and to the observation noise. The highlight of that measurement model was to do away with the undesired linearization procedure but it had the drawback of an increased, four-dimensional (4D), measurement model, where the measurement matrix was rank-degenerated.

This work builds on the foregoing efforts and suggests new developments for a family of more efficient continuous-discrete quaternion Kalman filters. It describes the Lyapunov exact differential equation for the true quaternion second moment. This equation has the nice property of preserving the unit-norm of the true quaternion in the mean-square sense. As a result, it allows an exact covariance propagation in the filter in spite of the multiplicative process noise. Further, the spectral properties of the quaternion measurement matrix, associated with a line-of-sight measurement [6], are described and exploited in order to develop several computationally efficient quaternion filters, while preserving the statistical information in some sense. Hinging on the rank-two degeneracy of the $4 \times 4$ measurement $H$-matrix, three different approaches are suggested in order to select an efficient $2 \times 4$ measurement matrix. The first method heuristically exploits a well-known performance criteria based on the Fisher information matrix $[7$, p. 84$]$ while the second method minimizes the trace of a state-dependent noise covariance matrix. Both methods aim at identifying the statistically most efficient $2 \times 4$ submatrix. The third method, however, constructs the two rows of a $2 \times 4$ matrix based on the two orthonormal basis vectors of the orthogonal complement to the Null-space of the $H$-matrix. These approaches lend themselves to different measurement update stages in novel continuous-discrete quaternion filters.

The baseline attitude determination system on-board Delfi-n3Xt includes a three-axis magnetometer, 6 body-mounted four-quadrant Sun sensors, and a 
three-axis rate gyroscope. While the magnetometer and the gyroscope are being purchased, the Sun sensors original design is begin carried out at TU Delft Chair of Space Systems Engineering. This paper describes ongoing developments of the Sun vector determination system. For each Sun sensor, the Sun line-ofsight is determined by a simple algorithm that does away with some uncertain physical parameters. The proposed algorithm takes into account geometrical imperfections due to manufacturing limitations. A thorough analysis of the errors associated with the photodiodes' measurements is presented, and approaches for Sun vector determination at spacecraft level, i. e., merging the outputs of the 6 Sun sensors, are proposed and illustrated via Monte-Carlo simulations and experimental validation. Finally, a numerical example of attitude determination using rate gyro, Sun vector, and Earth magnetic field measurements is presented illustrating via extensive simulations the performances of the novel quaternion filters. The novel filter paradigm shows a clear advantage with respect to a standard additive Kalman filter, which fails to converge in a rapidly tumbling dynamics environment.

Section 2 is devoted to the mathematical modeling, including the analysis of the Lyapunov differential equation of the quaternion second moment, the properties of the $H$-matrix, and the approaches for reducing the measurement model. Section 3 presents the Sun vector determination system design. Section 4 presents the numerical simulations of the attitude estimators. Conclusions are drawn in section 5 .

\section{MATHEMATICAL MODELING}

\subsection{Lyapunov Differential Equation for the Quaternion Second Moment}

It was shown in $[5,8]$ that the quaternion process equation can be expressed in the framework of Mean-Square calculus via the following Itô stochastic differential equation:

$$
d \mathbf{q}_{\mathbf{t}}=F_{I} \mathbf{q}_{\mathbf{t}} d t-\frac{1}{2} \Xi\left(\mathbf{q}_{\mathbf{t}}\right) d \beta_{\mathrm{t}} ; \mathbf{q}_{\mathbf{t}}(0) \stackrel{\text { a.e. }}{=} \mathbf{q}_{0} ; t \in[0, T]
$$

with

$$
F_{I}=\frac{1}{2}\left(\Omega_{t}-\frac{3 \sigma_{\epsilon}^{2}}{4} I_{4}\right)
$$

where $\Omega_{t}$ is the function of the measured angular velocity, $\Omega_{t}$, which is corrupted by an additive Brownian motion noise, $\beta_{t}$, and $d \beta_{t}$ denotes its infinitesimal independent increments such that $\mathrm{E}\left\{\beta_{t} \beta_{t}^{\mathrm{T}}\right\}=\sigma_{\varepsilon} I_{3} \delta(t-\tau)$. A known characteristic 
of this process equation is the state-multiplicative process noise where the noise input matrix, $\Xi$, is a known linear matrix function of the quaternion, $\mathbf{q}=\left[e^{\mathrm{T}} q\right]^{\mathrm{T}}$, i. e.,

$$
\Xi(\mathbf{q})=\left[\begin{array}{c}
q I_{3}-[e \times] \\
e^{\mathrm{T}}
\end{array}\right]
$$

where $[e \times]$ denotes the cross-product matrix, which is defined as follows: for any pair of vectors in $\mathbb{R}^{3}, \mathbf{x}$ and $\mathbf{y},[\mathbf{x} \times] \mathbf{y}=\mathbf{x} \times \mathbf{y}$. As a result, the Lyapunov differential equation governing the time propagation of the second moment of $\mathbf{q}_{\mathbf{t}}, X_{t}$, is expressed as follows:

$$
\dot{X}_{t}=F_{I} X_{t}+X_{t} F_{I}^{\mathrm{T}}+\frac{\sigma_{\epsilon}^{2}}{4}\left[\left(\operatorname{tr} X_{t}\right) I_{4}-X_{t}\right] ; X(0)=X_{0} .
$$

The damping terms on the main diagonal of $F_{I}$ allows for the process to be converging in the mean-square sense (m.s.s.), which ensures, in particular, the invariance of the quaternion norm (m.s.s.), i.e.,

$$
\mathrm{E}\left\{\left\|\mathbf{q}_{\mathbf{t}}\right\|^{2}\right\}=\operatorname{tr} X_{t}=\operatorname{tr} X_{0} \quad \forall t \geq 0 .
$$

The unit-norm property of $\mathbf{q}_{\mathbf{t}}$ can, thus, be preserved (m.s.s.) by adequately choosing the trace of $X_{0}$ equal to one.

Proposition 1. Consider the Lyapunov differential equation satisfied by the second-order moment of the quaternion, $X_{t}$, which is given in Eq. (3), and let $\mathbf{x}$ denote the $16 \times 1$ column-vector vec $X_{t}$, which is formed by stacking the columns of $X_{t}$ one onto the other, then

(i) the $4 \times 4$ matrix equation (3) is equivalent to the following 16-dimensional vector equation:

$$
\dot{\mathbf{x}}_{t}=\mathcal{A} \mathbf{x}_{t} ; \mathbf{x}(0)=\operatorname{vec} X_{0}
$$

where

$$
\begin{aligned}
\mathcal{A} & =\frac{1}{2}(\Omega \oplus \Omega)+\sigma_{\epsilon}^{2}\left(\hat{\mathbf{a}} \hat{\mathbf{a}}^{T}-I_{16}\right) ; \\
\Omega \oplus \Omega & =\Omega \otimes I_{4}+I_{4} \otimes \Omega ; \\
\hat{\mathbf{a}} & =\frac{1}{2} \operatorname{vec} I_{4}=\frac{1}{2}[1 \cdots 1 \cdots 1 \cdots 1]^{\mathrm{T}}
\end{aligned}
$$

and $\otimes$ denotes the Kronecker product; $I_{16}$ is the identity matrix in $\mathbb{R}_{16}$; and the '.' denote zeros in Eq. (4);

(ii) the spectrum of the matrix $\mathcal{A}$ is as follows:

$$
\operatorname{Sp}\{\mathcal{A}\}=\left\{0,-\sigma_{\epsilon}^{2(7)},-\sigma_{\epsilon}^{2}+j \omega^{(4)},-\sigma_{\epsilon}^{2}-j \omega^{(4)}\right\}
$$


where the superscript numbers in parentheses denote the algebraic multiplicity of the associated eigenvalues. Moreover, the kernel of $\mathcal{A}$ is generated by $\hat{\mathbf{a}}$ and the unique steady-state solution to Eq. (3) is as follows: $X_{\infty}=(1 / 4) I_{4} ;$ and

(iii) consider the case where the angular velocity vector is constant, then

$$
\begin{aligned}
\mathbf{x}_{t} & =e^{\mathcal{A} t} \mathbf{x}(0) \\
e^{\mathcal{A} t} & =\left[e^{(1 / 2) \Omega t} \otimes e^{(1 / 2) \Omega t}\right]\left[\hat{\mathbf{a}} \hat{a}^{\mathrm{T}}+e^{-\sigma_{\epsilon}^{2} t}\left(I_{16}-\hat{\mathbf{a}} \hat{\mathbf{a}}^{\mathrm{T}}\right)\right] .
\end{aligned}
$$

The proofs are straightforward and are not shown here for the sake of brevity.

Remark 1. The second bracket parentheses in the right-hand side of Eq. (5) provide an analytical expression for the influence of the state-multiplicative noise in Eq. (1) onto the quaternion second moment. Notice that the matrix in these brackets converges to the dyadic matrix ( $\hat{\mathbf{a}} \hat{\mathbf{a}}^{\mathrm{T}}$ ), as $t$ goes to $\infty$, which has the effect of orthogonally projecting the state vector $\mathbf{x}$ onto the vector line generated by the unit-vector $\hat{\mathbf{a}}$. Clearly, for $\sigma_{\epsilon}=0$ (no process noise), the solution for $X$ boils down to the solution of the standard Lyapunov equation, $\dot{X}=(1 / 2) \Omega X$ $+X(1 / 2) \Omega^{\mathrm{T}}$, which admits the following closed-form solution, for the case of a constant vector $\omega$ :

$$
X_{t}=e^{(1 / 2) \Omega t} X_{0} e^{(1 / 2) \Omega^{\mathrm{T}} t}
$$

When $\sigma_{\epsilon} \neq 0$, it will be shown that $X_{t} \sim(1 / 4) I_{4}$ when $t \sim \infty$.

Remark 2. If $\operatorname{tr} X_{0}=1$, then the steady-state is simply $\mathbf{x}_{\infty}=(1 / 2) \hat{\mathbf{a}}$, i.e., $X_{\infty}=(1 / 4) I_{4}$, which has the following interpretation: since the quaternion process is being driven by a Brownian motion that uniformly diffuses in its three directions, then the steady-state uncertainty in $\mathbf{q}_{\mathbf{t}}$ becomes equal in all four components.

\subsection{Properties of the Quaternion Measurement Matrix}

Let $\mathbf{b}$ and $\mathbf{r}$ denote the projections of an ideal noise-free line-of-sight measurement, also called vector measurement, onto the spacecraft coordinates frame, $\mathcal{B}$, and onto some reference frame, $\mathcal{R}$, respectively. It was shown [6] that the rotation quaternion from $\mathcal{R}$ to $\mathcal{B}$, q, belongs to the Kernel of a specific matrix, denoted by $H$, i. e.,

$$
H \mathbf{q}=\mathbf{0}
$$


where $H$ is computed using the pair $(\mathbf{b}, \mathbf{r})$ as follows:

$$
\begin{aligned}
\mathbf{s} & =\frac{1}{2}(\mathbf{b}+\mathbf{r}) \\
\mathbf{d} & =\frac{1}{2}(\mathbf{b}-\mathbf{r}) \\
H & =\left[\begin{array}{rr}
-[\mathbf{s} \times] & \mathbf{d} \\
-\mathbf{d}^{\mathrm{T}} & 0
\end{array}\right] .
\end{aligned}
$$

The following proposition states some insightful properties of the $H$-matrix.

Proposition 2. Given the pair of unit-norm column-vectors, $(\mathbf{b}, \mathbf{r})$, that are associated with a single vector observation then,

(i) the spectrum of $H$ is as follows:

$$
\operatorname{Sp} H=\{0,0, j,-j\}
$$

and there exist an orthonormal matrix, $Q \in \mathbb{R}^{4 \times 4}$, and a block-diagonal matrix, $\Lambda \in \mathbb{R}^{4 \times 4}$, such that

$$
H=Q \Lambda Q^{\mathrm{T}}=\left[\begin{array}{llll}
\mathbf{q}_{1} & \mathbf{q}_{2} & \mathbf{q}_{3} & \mathbf{q}_{4}
\end{array}\right]\left[\begin{array}{cccc}
0 & 1 & 1 & 1 \\
-1 & 0 & 1 & 1 \\
-1 & -1 & 0 & 1 \\
-1 & -1 & -1 & 0
\end{array}\right]\left[\begin{array}{l}
\mathbf{q}_{1}^{\mathrm{T}} \\
\mathbf{q}_{2}^{\mathrm{T}} \\
\mathbf{q}_{3}^{\mathrm{T}} \\
\mathbf{q}_{4}^{\mathrm{T}}
\end{array}\right]
$$

(ii) the kernel of $H$, Ker $H$, is generated by the orthonormal basis $\left\{\mathbf{q}_{1}, \mathbf{q}_{2}\right\}$ where

$$
\begin{aligned}
& \mathbf{q}_{1}=\left[\begin{array}{l}
\mathbf{s} \\
0
\end{array}\right] \frac{1}{\|\mathbf{s}\|} ; \\
& \mathbf{q}_{2}=\left[\begin{array}{c}
-\mathbf{s} \times \mathbf{d} \\
\|\mathbf{s}\|^{2}
\end{array}\right] \frac{1}{\|\mathbf{s}\|} ;
\end{aligned}
$$

(iii) the orthogonal complement of $\operatorname{Ker} H,(\operatorname{Ker} H)^{\perp}$, is generated by the orthonormal basis $\left\{\mathbf{q}_{3}, \mathbf{q}_{4}\right\}$ where

$$
\begin{aligned}
& \mathbf{q}_{3}=\left[\begin{array}{l}
\mathbf{d} \\
0
\end{array}\right] \frac{1}{\|\mathbf{d}\|} ; \\
& \mathbf{q}_{4}=\left[\begin{array}{l}
\mathbf{s} \times \mathbf{d} \\
\|\mathbf{d}\|^{2}
\end{array}\right] \frac{1}{\|\mathbf{d}\|} .
\end{aligned}
$$


The proofs hinge on classical results in matrix analysis and are omitted for the sake of brevity.

Remark 1. The vector $\mathbf{q}_{1}$ is a quaternion representing a $180^{\circ}$-rotation around the Euler axis supported by the vector s. Geometrically, this rotation is materialized by letting the tip of the $\mathbf{b}$-vector describe half-a-circle around $\mathbf{s}$ while staying in a plane that is orthogonal to the $(\mathbf{b}, \mathbf{r})$-plane. On the other hand, the vector $\mathbf{q}_{2}$ describes a minimal-angle rotation, and it corresponds to the Euler axis supported by the vector $\mathbf{s} \times \mathbf{d}$. In this case, the $\mathbf{b}$-vector is rotated until it coincides with the $\mathbf{r}$-vector, while staying in the $(\mathbf{b}, \mathbf{r})$-plane. The angle of the $\mathbf{q}_{2}$-rotation, $\varphi_{2}$, is easily determined from the scalar component of $\mathbf{q}_{2}$, i. e.,

$$
\varphi_{2}=2 \arccos (\|\mathbf{s}\|)
$$

Remark 2. The vectors $\mathbf{q}_{3}$ and $\mathbf{q}_{4}$ may also be interpreted as quaternions of rotation. In this case, however, the geometrically transformation maps $\mathbf{b}$ to $(-\mathbf{r})$ instead of $\mathbf{r}$. The $\mathbf{q}_{3}$-rotation is, thus, a $180^{\circ}$-rotation around the Euler axis supported by $\mathbf{d}$, where the tip of the transformed vector describes half-a-circle, from $\mathbf{b}$ to $(-\mathbf{r})$, in the plane orthogonal to $(\mathbf{b}, \mathbf{r})$. The $\mathbf{q}_{4}$-rotation brings $\mathbf{b}$ to $(-\mathbf{r})$ by rotating the transformed vector in the plane $(\mathbf{b}, \mathbf{r})$ and around the axis supported by $\mathbf{s} \times \mathbf{d}$. The Euler angle of that rotation is simply the supplementary angle to $\varphi_{2}$, as given in Eq. (11).

\subsection{Reduced Quaternion Measurement Model}

Consider the four dimensional quaternion measurement model that was introduced in [6], i.e.,

$$
\mathbf{0}=H_{k} \mathbf{q}_{k}-\frac{1}{2} \Xi_{k} \boldsymbol{\delta} b_{k}
$$

where $H_{k}$ is defined in Eq. (8) using the noisy vector $\mathbf{b}_{k}, \boldsymbol{\delta} b_{k}$ is an additive zeromean white noise with covariance matrix $R_{k}$, and $\Xi_{k}$ denotes the quaterniondependent $4 \times 3$ matrix, as defined in Eq. (2). Since a $4 \mathrm{D} H$-matrix that is computed from a single vector observation is of rank two, then a two-dimensional (2D) measurement model can be developed and used in a quaternion filter. The measurement update stage in that filter will, thus, have a reduced computational burden. Three possible approaches for the desired model reduction will be described next. The first two approaches suggest extracting a $2 \times 4$ submatrix of $H$ according to simple, intuitive, rationales. The third approach hinges on the spectral properties of the $H$-matrix in order to construct an adequate $2 \times 4$ measurement equation. 


\subsubsection{Approach \#1}

There are six $2 \times 4$ submatrices of $H_{k}$, each one being of rank two. They correspond to the following pairs of rows: $(1,2),(1,3),(1,4),(2,3),(2,4)$, and $(3,4)$. Their expressions, denoted by $H^{i j}$, are provided in Table 1 . From an algebraic point of view, all six reduced measurement matrices are equivalent. In the context of stochastic filtering, however, the particular choice of one of these matrices may impact the statistical properties of the estimation process, as will be pointed out next, and, thus, provide the user with a meaningful criterion for picking up the right model. The heuristics stems from the concept of the Fisher information matrix and on the following known result (see, e.g., [7, p. 84]): Assuming a standard linear measurement model, i. e., $\mathbf{z}_{k}=M_{k} \theta+\mathbf{v}_{k}$ where $M_{k}$ and $\theta$ are deterministic and $\mathbf{v}_{k}$ is a vector of zero-mean independently identically distributed Gaussian noises, with variance $\sigma^{2}$, then the Fisher information matrix, $J(\theta)$, associated with the measurement $\mathbf{z}_{k}$, is expressed as

$$
J(\theta)=\frac{1}{\sigma^{2}} M_{k}^{\mathrm{T}} M_{k}
$$

Hinging on the above result, the proposed method for choosing the reduced measurement matrix consists in maximizing some measure of the matrix $J$. Since $J$ is positive semidefinite, one can use the value of its trace, which is proportional to the squared Frobenius norm of the associated measurement matrix. The

Table 1 Six possible reduced quaternion measurement matrices and their performance index

\begin{tabular}{cccc}
\hline Model & Rows & $H^{i j}$ & $\left\|H^{i j}\right\|_{F}^{2}$ \\
\hline 1 & $(1,2)$ & {$\left[\begin{array}{cccc}0 & s_{3} & -s_{2} & d_{1} \\
-s_{1} & 0 & s_{1} & d_{2}\end{array}\right]$} & $1+b_{3} r_{3}$ \\
2 & $(1,3)$ & {$\left[\begin{array}{cccc}0 & s_{3} & -s_{2} & d_{1} \\
s_{2} & -s_{1} & 0 & d_{3}\end{array}\right]$} & $1+b_{2} r_{2}$ \\
3 & $(1,4)$ & {$\left[\begin{array}{cccc}0 & s_{3} & -s_{2} & d_{1} \\
-d_{1}-d_{2} & -d_{3} & 0\end{array}\right]$} & $1-b_{1} r_{1}$ \\
4 & $(2,3)$ & {$\left[\begin{array}{cccc}-s_{3} & 0 & s_{1} & d_{2} \\
s_{2} & -s_{1} & 0 & d_{3}\end{array}\right]$} & $1+b_{1} r_{1}$ \\
5 & $(2,4)$ & {$\left[\begin{array}{cccc}-s_{3} & 0 & s_{1} & d_{2} \\
-d_{1}-d_{2} & -d_{3} & 0\end{array}\right]$} & $1-b_{2} r_{2}$ \\
6 & $(3,4)$ & {$\left[\begin{array}{cccc}s_{2} & -s_{1} & 0 & d_{3} \\
-d_{1}-d_{2}-d_{3} & 0\end{array}\right]$} & $1-b_{3} r_{3}$ \\
\hline
\end{tabular}


Frobenius norms of the six matrices $H^{i j}$, i.e.,

$$
\left\|H^{i j}\right\|_{F}=\sqrt{\operatorname{tr}\left[\left(H^{i j}\right)^{\mathrm{T}} H^{i j}\right]},
$$

are easily computed and are provided in Table 1, as functions of the components of the vectors $\mathbf{b}$ and $\mathbf{r}$.

Method: For each acquired vector observation, $(\mathbf{b}, \mathbf{r})$, compute the $H$-matrix, as given in Eq. (8), and compute the performance index $\left\|H^{i j}\right\|_{F}^{2}$ (see Table 1) for the six possible reduced models. Then choose, out of the six possible models, the model that maximizes the performance.

\subsubsection{Approach \#2}

The second approach also hinges on a statistical rationale, but only considers the covariance matrix of the associated state-dependent measurement noise. Consider the $4 \mathrm{D}$ quaternion measurement model (12). Let $P_{k}^{v}$ denote the covariance matrix of the quaternion-dependent noise appearing in Eq. (12), i. e.,

$$
P_{k}^{v} \triangleq \operatorname{cov}\left\{-\frac{1}{2} \Xi_{k} \boldsymbol{\delta} b_{k}\right\} .
$$

This matrix can be exactly expressed as a function of the first two moments of $\mathbf{q}_{k}$ [6]. Reducing the measurement model (12) by simply extracting two rows obviously impacts the resulted measurement noise. For instance, choosing the pair of rows $(1,4)$ yields the following $2 \times 2$ reduced noise covariance matrix:

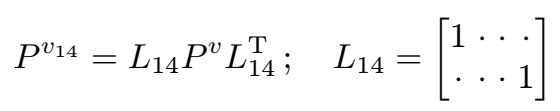

where the dots are representing zeros. A method is henceforth suggested for choosing the pair of rows according to the following rationale: the extracted pair will be such that the covariance of the associated reduced measurement noise will be minimal among all possible choices. The measure of performance will be the trace of the reduced noise covariance matrix.

Method for a general $\boldsymbol{P}_{\boldsymbol{v}}$. The method is summarized by the following steps. As a preliminary, let the six matrices $L_{i j}, i, j=1,2,3,4, j>i$, be defined as follows: any matrix $L_{i j}$ is a $2 \times 4$ matrix with all entries equal to zero except for the entries at $(1, i)$ and $(2, j)$, which are equal to one.

Step \#1. Compute the six possible $2 \times 2$ reduced noise covariance matrices, $\left\{P^{v_{i j}}\right\}$ :

$$
P^{v_{i j}}=L_{i j} P^{v} L_{i j}^{\mathrm{T}}
$$


Step \#2. Compute their traces:

$$
\alpha_{i j}=\operatorname{tr} P^{v_{i j}}
$$

Step \#3. Choose $(i, j)$ to solve $\min _{i, j} \alpha_{i j}$ and use, accordingly, the reduced matrices, $H^{i j}$ and $P^{v_{i j}}$, in the reduced measurement update stages of the quaternion filters.

Case A: $R_{k}=\sigma_{b}^{2} I_{3}$. The method that results for Case $\mathrm{A}$ is simple and is essentially summarized in Table 2. It can be shown [6] that, for such a matrix $R_{k}$,

$$
P_{k}^{v}=\frac{\sigma_{b}^{2}}{4}\left(I_{4}-X_{k}\right)
$$

where, between measurement epochs, $X_{t}$ can be propagated using Eq. (3). This yields the value of $X_{t}$ and, thus, of its diagonal elements, $x_{i i}$. Finding the pair $(i, j)$ that minimizes the criterion $\alpha_{i j}$, i. e., $\left(2-x_{i i}-x_{j j}\right)$, yields the most efficient reduced model. There is an intuitive interpretation to this selection method. Assume, for example, that the first and the forth components of $\mathbf{q}$, i. e., $e_{1}$ and $q$, have the highest second-order moments, $x_{11}$ and $x_{44}$, respectively. Since the quaternion components act as noise amplifiers in the noise multiplicative term, then the adequate model should be the one where neither $e_{1}$ nor $\mathbf{q}$ enter the expression for that multiplicative noise. This can be partly achieved by choosing the reduced matrix $H^{14}$, since the coefficients multiplying $e_{1}$ and $\mathbf{q}$ are equal to zero in the first and forth rows, respectively, as seen from the expression for $H^{14}$ :

$$
H^{14}=\left[\begin{array}{cccc}
0 & s_{3} & -s_{2} & d_{1} \\
-d_{1} & -d_{2} & -d_{3} & 0
\end{array}\right]
$$

Indeed, for this case, the proposed method selects the reduced matrix $H^{14}$.

Table 2 Six possible reduced noise covariance matrices and their performance index, for $i, j$ $=1,2,3,4, j>i, x_{i i}$ and $x_{j j}$ are the diagonal elements in the matrix $X_{t}$

\begin{tabular}{ccc}
\hline Rows & $\frac{4}{\sigma_{b}^{2}} P^{v_{i j}}$ & $\frac{4}{\sigma_{b}^{2}} \alpha_{i j}$ \\
\hline$(i, j)$ & {$\left[\begin{array}{cc}1-x_{i i} & -x_{i j} \\
-x_{i j} & 1-x_{j j}\end{array}\right]$} & $2-x_{i i}-x_{j j}$ \\
\hline
\end{tabular}


Case B: $\quad R_{k}=\sigma_{b}^{2}\left(I_{3}-\mathbf{b}_{k} \mathbf{b}_{k}^{\mathrm{T}}\right)$. The proposed matrix $R_{k}$ is a usual approximate expression for the covariance matrix of the unit-norm vector $\mathbf{b}_{k}$. It was shown [6] that, for such a matrix $R_{k}$,

$$
P^{v}=\frac{\sigma_{b}^{2}}{4}\left(I_{4}-X-B X B^{\mathrm{T}}\right)
$$

where the time subscript was dropped for simplicity, and

$$
B=\left[\begin{array}{cc}
-[\mathbf{b} \times] & \mathbf{b} \\
-\mathbf{b}^{\mathrm{T}} & 0
\end{array}\right] .
$$

The method for Case B is summarized next.

Step \#1. Compute the three scalar quantities, $\beta_{i}, i=1,2,3$ :

$$
\begin{aligned}
& \beta_{1}=\left(1-b_{1}^{2}\right)\left(-x_{11}+x_{22}+x_{33}-x_{44}\right)+2\left[b_{1} b_{2}\left(x_{12}-x_{34}\right)+b_{1} b_{3}\left(x_{13}+x_{24}\right)\right] \\
& \beta_{2}=\left(1-b_{2}^{2}\right)\left(x_{11}-x_{22}+x_{33}-x_{44}\right)+2\left[b_{1} b_{2}\left(x_{12}+x_{34}\right)+b_{2} b_{3}\left(x_{23}-x_{14}\right)\right] \\
& \beta_{3}=\left(1-b_{3}^{2}\right)\left(x_{11}+x_{22}-x_{33}-x_{44}\right)+2\left[b_{1} b_{3}\left(x_{13}-x_{24}\right)+b_{2} b_{3}\left(x_{23}+x_{14}\right)\right]
\end{aligned}
$$

where the scalars $x_{i j}$ are the entries of $X$.

Step \#2. Solve

$$
\beta^{*}=\min \left(\beta_{1},-\beta_{1}, \beta_{2},-\beta_{2}, \beta_{3},-\beta_{3}\right)
$$

and choose the adequate pair of rows according to the relationships shown in Table 3 . The above steps are obtained by applying the general method previously described. Once the adequate model is identified from Table 3, the corresponding reduced order matrix, $H^{i j}$, may be obtained from Table 1 , and the associated reduced noise covariance matrix can be computed using Eq. (13). Notice that $R_{k}$ is of rank two, and so is the reduced matrix $P^{v_{i j}}$.

\subsubsection{Approach \#3}

Another approach for reducing the quaternion measurement model, as given in Eq. (12), consists in exploiting the spectral properties of the $H$-matrix, as provided in Eqs. (9) and (10), i. e., in using the closed-form expression for the orthonormal basis, $\left\{\mathbf{q}_{3}, \mathbf{q}_{4}\right\}$, of $(\operatorname{Ker} H)^{\perp}$.

Proposition 3. Given a single vector observation, $\left(\mathbf{b}_{k}, \mathbf{r}_{k}\right)$ where $\mathbf{b}_{k}$ is corrupted by an additive zero-mean white noise $\delta b_{k}$ with covariance matrix $R_{k}$, then, 
(i) the quaternion, $\mathbf{q}_{k}$, satisfies the following reduced $2 \times 1$ quaternion measurement equation:

$$
\mathbf{0}=\bar{H}_{k} \mathbf{q}_{k}+\overline{\mathbf{v}}_{k}
$$

where the $2 \times 2$ matrix $\bar{H}_{k}$ is computed as follows:

$$
\bar{H}_{k}=\left[\begin{array}{cc}
\left(\mathbf{s}_{k} \times \mathbf{d}_{k}\right)^{\mathrm{T}} & \left\|\mathbf{d}_{k}\right\|^{2} \\
-\mathbf{s}_{k}^{\mathrm{T}} & 0
\end{array}\right] \frac{1}{\left\|\mathbf{d}_{k}\right\|}
$$

with $\mathbf{s}$ and $\mathbf{d}$ as given in Eqs. (6) and (7), and $\overline{\mathbf{v}}_{k}$ is defined as the following state-dependent noise:

$$
\begin{aligned}
& \overline{\mathbf{v}}_{k}=-\frac{1}{2} \bar{V}_{k} \Xi\left(\mathbf{q}_{k}\right) \boldsymbol{\delta} b_{k} ; \\
& \bar{V}_{k}=\left[\begin{array}{cc}
\mathbf{s}_{k}^{\mathrm{T}} & 0 \\
\left(\mathbf{s}_{k} \times \mathbf{d}_{k}\right)^{\mathrm{T}} & \left\|\mathbf{d}_{k}\right\|^{2}
\end{array}\right] \frac{1}{\left\|\mathbf{d}_{k}\right\|}
\end{aligned}
$$

Table 4 Reduced multiplicative noise covariance matrix, $P_{k}^{\bar{v}}$, and its steady-state for two cases of $R_{k}$

\begin{tabular}{ccc}
\hline$R_{k}$ & $P_{k}^{\bar{v}}$ & $P_{\infty}^{\bar{v}}$ \\
\hline$\sigma_{b}^{2} I_{3}$ & $\frac{1}{4} \sigma_{b}^{2}\left(I_{2}-\bar{V}_{k} X_{k} \bar{V}_{k}^{\mathrm{T}}\right)$ & $\frac{3}{16} \sigma_{b}^{2} I_{2}$ \\
$\sigma_{b}^{2}\left(I_{3}-\mathbf{b}_{k} \mathbf{b}_{k}^{\mathrm{T}}\right)$ & $\frac{1}{4} \sigma_{b}^{2}\left(I_{2}-\bar{V}_{k} X_{k} \bar{V}_{k}^{\mathrm{T}}-\bar{C}_{k} X_{k} \bar{C}_{k}^{\mathrm{T}}\right)$ & $\frac{1}{8} \sigma_{b}^{2} I_{2}$ \\
\hline
\end{tabular}

(ii) the measurement noise, $\overline{\mathbf{v}}_{k}$, is a white noise sequence with zero mean (to first order in $\|\boldsymbol{\delta} \boldsymbol{b}\|$ ) and with a second moment, $P^{\bar{v}}$, which is next expressed, as a function of $X_{k}$, for specific but important cases of $R_{k}$, in Table 4 where $\bar{V}_{k}$ is given in Eq. (14) and the $2 \times 4$ matrix $\bar{C}_{k}$ is computed as follows:

$$
\bar{C}_{k}=\left[\begin{array}{cc}
\mathbf{r}_{k}^{\mathrm{T}}\left[\mathbf{b}_{k} \times\right] & 1-\mathbf{r}_{k}^{\mathrm{T}} \mathbf{b}_{k} \\
\frac{1}{2} \mathbf{r}_{k}^{\mathrm{T}}\left(I_{3}+3 \mathbf{b}_{k} \mathbf{b}_{k}^{\mathrm{T}}\right)-2 \mathbf{b}_{k}^{\mathrm{T}} & 0
\end{array}\right] \frac{1}{\sqrt{2} \sqrt{1-\mathbf{b}_{k}^{\mathrm{T}} \mathbf{r}_{k}}} .
$$

The proof of Proposition 3 is straightforward.

\subsection{Concluding Remarks}

Each of the above approaches for reduction of the quaternion measurement model lends itself to a specific measurement update stage in the Kalman filter. These 
stages are of dimension two, which lowers the computational burden in the filter, as compared to the $4 \mathrm{D}$ stage designed in [8]. The provision of analytical expressions for the minimization criteria, and for the covariance matrices, balances the need for additional algebraic manipulations. The resulting Kalman filters are straightforward to develop and their expressions are omitted here for the sake of brevity.

\section{SUN VECTOR DETERMINATION SYSTEM}

The Sun vector determination on board Delfi-n3Xt involves a system of six Sun sensors, one on each of the six body panels of the three-units CubeSat. This configuration was chosen in order to ensure that the Sun will be visible independent of the satellite's attitude. This requirement is critical for mission success where the aim is to demonstrate three-axis Sun pointing stability. The Sun sensors were mounted on the body because the entire area of the solar panels was needed for power generation.

\subsection{Sensor Sun Vector Determination}

The subsequent analysis focuses on a single Sun sensor. The Sun sensor that is designed is of the quadrant type, where four similar square photodiodes are tightly located next to each together in a square formation. The photodiodes can absorb light, which passes through a square hole that has the same dimensions as each photodiode. Depending on the direction of the incoming light, the area that is lit moves over the four quadrants and a photocurrent is being generated and measured for each of the quadrants. As can be seen from Fig. 1a, the lit area on each photodiode is direction dependent. This dependency between the sensor orientation and the measured currents stands at the heart of the operation principle of the Sun sensor. It should be already noted that a practical design has to take into account various parameters such as geometrical constraints like the angular divergence of the incoming light, manufacturing constraints like blunt edges, and disturbances due to measurement random noises or perturbations induced by secondary light sources like the Earth albedo. Part of the design developments taking into account these aspects will be presented in the following.

Let focus next on the ideal case in order to develop the basic Sun vector determination algorithm for a single Sun sensor. Let $\left\{v_{i}, i=1,2,3\right\}$ denote the components of the Sun unit vector, $\mathbf{v}$, along the Sun sensor axes. Let $\alpha$ and $\beta$ be defined as in Fig. 1a. They are related to the components $v_{i}$ as follows:

$$
\tan \alpha=\frac{v_{1}}{v_{3}} ; \quad \tan \beta=\frac{v_{2}}{v_{3}} .
$$




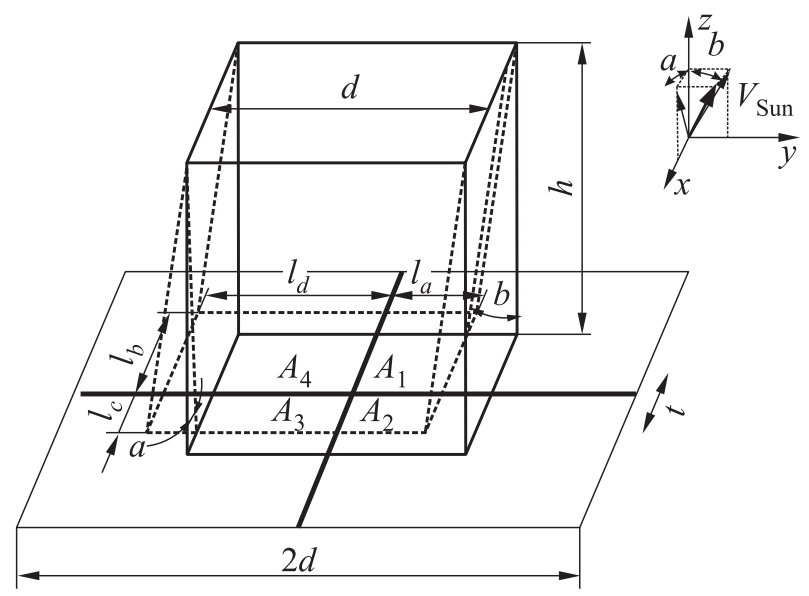

(a)

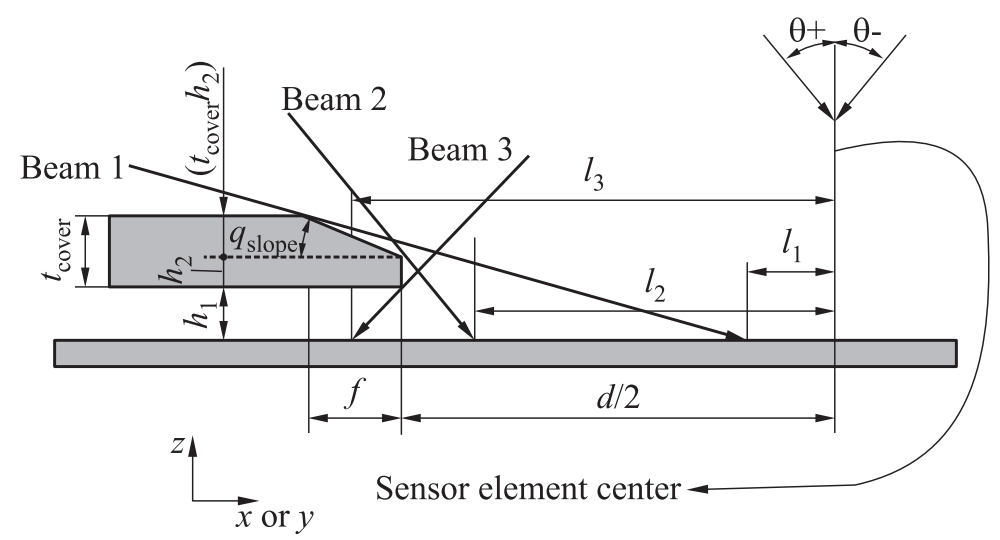

(b)

Figure 1 Sun sensor geometry: (a) four-quadrant Sun sensor; and (b) cross section with cover dimensions

Henceforth, the expression for the unit Sun vector $\mathbf{v}$ is as follows:

$$
\mathbf{v}=\frac{1}{\sqrt{1+\tan ^{2} \alpha+\tan ^{2} \beta}}\left[\begin{array}{c}
\tan \alpha \\
\tan \beta \\
1
\end{array}\right]
$$




\subsubsection{Ideal case}

Equation (15) shows how to compute the vector $\mathbf{v}$ for given values of $\tan \alpha$ and $\tan \beta$. As depicted in Fig. $1 a$, these quantities are related to the lit areas of the four-quadrant Sun sensor, which are themselves functions of the photocurrent intensities at each diode, $I_{i}, i=1,2,3,4$.

The proposed approach computes two ratios, $R_{1}$ and $R_{2}$, which are the observables, from which the two unknowns, $\tan \alpha$ and $\tan \beta$, and thus, the sensor Sun vector, can be determined, as described next. Given acquired measurements of the photodiodes currents, $I_{i}, i=1,2,3,4$,

$$
\begin{aligned}
R_{1} & =\frac{I_{1}+I_{2}-I_{3}-I_{4}}{I_{1}+I_{2}+I_{3}+I_{4}} ; \\
R_{2} & =\frac{I_{3}+I_{2}-I_{1}-I_{4}}{I_{1}+I_{2}+I_{3}+I_{4}} ; \\
\tan \alpha & =-\frac{2 h}{d-t} R_{2} ; \\
\tan \beta & =-\frac{2 h}{d-t} R_{1} ; \\
\widehat{\mathbf{v}} & =\frac{1}{\sqrt{1+\tan ^{2} \alpha+\tan ^{2} \beta}}\left[\begin{array}{c}
\tan \alpha \\
\tan \beta \\
1
\end{array}\right] .
\end{aligned}
$$

The above algorithm was developed under assumptions of zero Sun divergence, no manufacturing imperfections, and an ideal linear relationship between illuminated areas and photodiodes current intensity. Note that the coefficients of proportionality are usually uncertain physical parameters.

By doing away with these factors, the proposed approach is simpler and less subject to errors than an approach involving individual current-orientation relationships.

\subsubsection{Manufacturing imperfections}

In a physical implementation of the quadrant sensor, the hole is made in a cover above the sensor. Since the cover has a certain thickness, this influences the lengths $l_{a}, l_{b}, l_{c}$, and $l_{d}$.

Let $\theta$ denote the incident light angle, as depicted in Fig. $1 b$. The geometrical characteristics of the cover near the hole induce constraints on the incoming rays as depicted by the three beams in Fig. 1b. A straightforward analysis yields 


$$
l= \begin{cases}\frac{d}{2}-\frac{t}{2}-h_{1} \tan \theta & \text { if } \theta \leq 0 \\ \frac{d}{2}-\frac{t}{2}-\left(h_{1}+h_{2}\right) \tan \theta & \text { if } 0 \leq \theta \leq \frac{\pi}{2}-\theta_{\text {slope }} \\ \frac{d}{2}-\frac{t}{2}+\frac{t_{\text {cov }}-h_{2}}{\tan \left(\theta_{\text {slope }}\right)}-\left(h_{1}+t_{\text {cov }}\right) \tan \theta & \text { if } \theta \geq \frac{\pi}{2}-\theta_{\text {slope }}\end{cases}
$$

Using Eq. (18), it is possible to derive expressions for $l_{a}, l_{b}, l_{c}$, and $l_{d}$. Using a similar method as in the previous paragraph yields the relevant expressions for the sought quantities, $\tan \alpha$ and $\tan \beta$, as functions of the ratios $R_{1}, R_{2}$, and of the geometrical parameters defined in Figs. $1 a$ and $1 b$. The Sun vector determination algorithm for a single Sun sensor is summarized as follows. Given acquired measurements of the photodiodes currents, $I_{i}, i=1,2,3,4$,

$$
\begin{aligned}
& R_{1}= \frac{I_{1}+I_{2}-I_{3}-I_{4}}{I_{1}+I_{2}+I_{3}+I_{4}} ; \\
& R_{2}= \frac{I_{3}+I_{2}-I_{1}-I_{4}}{I_{1}+I_{2}+I_{3}+I_{4}} ; \\
& \tan \alpha= \begin{cases}-\frac{R_{2}[d-t+f]+f}{2 h_{1}+t_{\mathrm{cov}}+R_{2} t_{\mathrm{cov}}} & R_{2} \geq-\frac{f\left(h_{2}+2 h_{1}\right)}{h_{2}(f+d-t)-t_{\mathrm{cov}}(d-t)} ; \\
-\frac{(d-t) R_{2}}{2 h_{1}+h_{2}+R_{2} h_{2}} & 0 \leq R_{2} \leq-\frac{f\left(h_{2}+2 h_{1}\right)}{h_{2}(f+d-t)-t_{\mathrm{cov}}(d-t)} \\
-\frac{(d-t) R_{2}}{2 h_{1}+h_{2}-R_{2} h_{2}} & \frac{f\left(h_{2}+2 h_{1}\right)}{h_{2}(f+d-t)-t_{\mathrm{cov}}(d-t)} \leq R_{2} \leq 0 ; \\
-\frac{R_{2}[d-t+f]-f}{2 h_{1}+t_{\mathrm{cov}}-R_{2} t_{\mathrm{cov}}} & R_{2} \leq \frac{f\left(h_{2}+2 h_{1}\right)}{h_{2}(f+d-t)-t_{\mathrm{cov}}(d-t)} ; \\
\tan \beta= & \begin{cases}-\frac{R_{1}[d-t+f]+f}{2 h_{1}+t_{\mathrm{cov}}+R_{1} t_{\mathrm{cov}}} & R_{1} \geq-\frac{f\left(h_{2}+2 h_{1}\right)}{h_{2}(f+d-t)-t_{\mathrm{cov}}(d-t)} ; \\
-\frac{(d-t) R_{1}}{2 h_{1}+h_{2}+R_{1} h_{2}} & 0 \leq R_{1} \leq-\frac{f\left(h_{2}+2 h_{1}\right)}{h_{2}(f+d-t)-t_{\mathrm{cov}}(d-t)} \\
-\frac{(d-t) R_{1}}{2 h_{1}+h_{2}-R_{1} h_{2}} & \frac{f\left(h_{2}+2 h_{1}\right)}{h_{2}(f+d-t)-t_{\mathrm{cov}}(d-t)} \leq R_{1} \leq 0 ; \\
-\frac{R_{1}[d-t+f]-f}{2 h_{1}+t_{\mathrm{cov}}-R_{1} t_{\mathrm{cov}}} & R_{1} \leq \frac{f\left(h_{2}+2 h_{1}\right)}{h_{2}(f+d-t)-t_{\mathrm{cov}}(d-t)} ;\end{cases} \end{cases}
\end{aligned}
$$




\subsubsection{Measurement errors}

A simplified measurement chain for each photodiode consists of a photodiode that converts light into current followed by an operational amplifier (op-amp), which output voltage is proportional to the input current, in series with an analog-to-digital converter (ADC) that provides a quantized signal at a specific sampling rate. As a result, the measured output voltage is expressed as follows

$$
\begin{aligned}
V_{0}=R_{f}\left(I_{S}+I_{\mathrm{dist}}-I_{d}-I_{\mathrm{sh}}+\right. & \left.I_{n, \mathrm{phot}}+I_{n-}\right) \\
& -\frac{1}{\beta} e_{n, \mathrm{amp}}-e_{n, f}+e_{\mathrm{qu}, \mathrm{ADC}}+e_{n, \mathrm{ADC}} .
\end{aligned}
$$

Here, $I_{S}$ denotes the current intensity due to the direct incident Sun light, and all other terms are disturbances and noises. Their source, type, and magnitudes are summarized in Table 5.

Let $V_{i}, i=1,2,3,4$, denote the noisy voltage outputs, as given in Eq. (24). These signals are produced onboard by the four photodiodes electronic assemblies of a single Sun sensor. Assuming a linear relationship between the output voltages and current intensities, with identical proportionality factor, these voltage values are used in Eqs. (19) and (20), instead of the currents intensities, i. e.,

Table 5 Summary of the Sun sensor signal and measurement errors in the voltage outputs

\begin{tabular}{llll}
\hline \multicolumn{1}{c}{ Signal } & \multicolumn{1}{c}{ Source } & \multicolumn{1}{c}{ Type } & Magnitude, V \\
\hline$R_{f} I_{S}$ & Sun & Signal & 2.64 \\
$R_{f} I_{\text {dist }}$ & Earth albedo & Bias & 0.88 \\
$R_{f} I_{d}$ & $\varphi$ dark current & Bias & $1.5 \cdot 10^{-8}$ \\
$R_{f} I_{s h}$ & $\varphi$ leak current & Bias & $3.9 \cdot 10^{-6}$ \\
$R_{f} I_{J}$ & $\varphi$ Johnson current noise & Gaussian & $4.8 \cdot 10^{-11}$ \\
$R_{f} I_{S, 0}$ & $\varphi$ signal current shot noise & Poisson & $3.4 \cdot 10^{-7}$ \\
$R_{f} I_{S, d}$ & $\varphi$ dark current shot noise & Poisson & $2.2 \cdot 10^{-11}$ \\
$R_{f} I_{S, \text { sh }}$ & $\varphi$ leak current shot noise & Poisson & $3.6 \cdot 10^{-10}$ \\
$R_{f} I_{n-}$ & Op-amp equivalent current noise & Gaussian & $4.8 \cdot 10^{-10}$ \\
$\beta^{-1} e_{n, \mathrm{amp}}$ & Op-amp equivalent voltage noise & Gaussian & $4.3 \cdot 10^{-7}$ \\
$e_{n, f}$ & Op-amp Johnson voltage noise & Gaussian & $3.9 \cdot 10^{-8}$ \\
$e_{\mathrm{qu}, \mathrm{ADC}}$ & ADC quantization noise & Uniform & $1.6 \cdot 10^{-5}$ \\
$e_{n, \mathrm{ADC}}$ & ADC noise & Gaussian & $1.1 \cdot 10^{-5}$ \\
\hline
\end{tabular}




$$
\begin{aligned}
& R_{1}=\frac{V_{1}+V_{2}-V_{3}-V_{4}}{V_{1}+V_{2}+V_{3}+V_{4}} \\
& R_{2}=\frac{V_{3}+V_{2}-V_{1}-V_{4}}{V_{1}+V_{2}+V_{3}+V_{4}}
\end{aligned}
$$

The remainder of the sensor Sun vector determination is carried out following Eqs. (21)-(23).

\subsection{Spacecraft Sun Vector Determination}

This subsection describes two algorithms used for merging the outputs of the Sun sensors in order to estimate the spacecraft Sun vector. The first algorithm is based on a constrained least-squares (LSQ) approach. The proposed problem is formulated as follows:

$$
\min _{\mathbf{v}^{\mathrm{T}} \mathbf{v}=1}\left[J(\mathbf{v})=\frac{1}{2} \sum_{j=1}^{6} w_{j}\left\|\mathbf{v}-\widehat{\mathbf{v}}_{j}\right\|^{2}\right]
$$

where $\mathbf{v}$ denotes the unit-norm spacecraft Sun vector, $\widehat{\mathbf{v}}_{j}$ denote the unit-norm sensor Sun vector estimates, as determined in Eq. (17), and $w_{j}$ are the positive scalar weights designed according to the relative reliability of each estimate. Notice that, by a slight abuse of notation, the vectors $\widehat{\mathbf{v}}_{j}$ in Eq. (26) represent the sensor Sun vectors along the spacecraft body frame (while the algorithms previously introduced compute their representation along each sensor coordinate frame). The problem of determining the misalignment between the sensor and the spacecraft body frames is not treated in this work. The solution to the problem (26) is easily shown to be as follows:

$$
\widehat{\mathbf{v}}_{\mathrm{SP}}=\frac{\sum_{j=1}^{6} w_{j} \widehat{\mathbf{v}}_{j}}{\left\|\sum_{j=1}^{6} w_{j} \widehat{\mathbf{v}}_{j}\right\|} .
$$

The weighting coefficients, $w_{j}$, are computed as follows:

$$
w_{j}=\sum_{i=1}^{4} V_{i, j}
$$

where $V_{i, j}$ denotes the voltage output from the photodiode $i$ at the sensor $j$. This choice relies on the heuristics that a sensor Sun vector estimate should be trusted more when the sensor is more illuminated and thus produces larger voltages. 
The second algorithm (HC) compares the values of the voltage signals at the six Sun sensors and selects the Sun vector estimate produced by the sensor with the maximum signal. The implementation is obviously simpler, and the results not so different from the previous algorithm, as will be shown next.

\subsection{Simulation Results}

A numerical simulation was performed providing the output voltages of all sensors for any Sun spacecraft $(\mathrm{S} / \mathrm{C})$ relative position. The influence of the Earth albedo was not accounted for in this investigation. On the other hand, the simulation accounts for the Sun angular divergence. This relies on the principle that the hole in the Sun sensor cover consists of the superposition of a very large number of smaller holes, each one diffracting the Sun rays onto the photodiodes. Thus, the validity of the zero angular divergence, on which the Sun vector determination solution relies, could be validated by simulation. The numerical values of the Sun sensor geometry (see Fig. 1) are provided in Table 6 . Figure $2 a$ shows the angular error between the true and the estimated spacecraft Sun vectors as a function of the incidence angle. These simulation results relate to the Sun vector determination algorithm with manufacturing imperfections and measurement errors. The largest error is about $0.3^{\circ}$ at around $50^{\circ}$. The spread of the different points at the same incidence angle stems from the randomness of the Monte-Carlo technique employed in simulating the Sun rays. The LSQ and the HC slgorithms were tested and compared. Figure $2 b$ shows the angular error between the true and the estimated Sun vector for 450 various incidence angles, varying from $0^{\circ}$ to $70^{\circ}$; similar performances with a slight advantage for the $\mathrm{HC}$ estimator. It has a lower maximum error, a lower average, and since it is simpler, a lower computation time. Further investigation is required in order to design statistically better estimators.

\subsection{Experimental Results}

A prototype of the Sun sensor was manufactured with the design parameters provided in Table 6 in order to validate experimentally the Sun vector determination approach. The experimental setup included a beamer as the light source. The "measured" ratio $R_{2}$ was computed as given in Eq. (25) using the measured output voltages, while a "predicted" ratio $R_{2}$ was computed using the model formula (16) between $R_{2}$ and the incidence angle $\alpha$. The results are shown in Fig. 3, which depicts a good fit between measurement and prediction. 




(a)

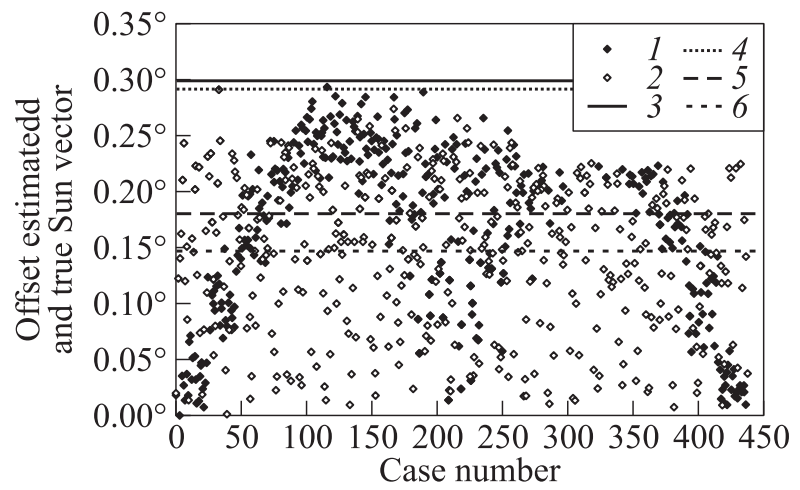

(b)

Figure 2 Angular error between the true and the estimated Sun vector as a function of the incidence angle: $(a)$ sensor Sun vector simulated with (1) and without (2) noise; and $(b)$ S/C Sun vector (1 - LSQ algorithm; $2-\mathrm{HC}$ algorithm; 3 and $5-$ maximum and average values (LSQ algorithm), respectively; and 4 and 6 - maximum and average values (HC algorithm), respectively)

\section{ATTITUDE DETERMINATION EXAMPLE}

In the proposed simulated scenarios, the CubeSat Delfi-n3Xt is orbiting around the Earth on a circular Sun-synchronous orbit at a height of $600 \mathrm{~km}$. Its principal body moments of inertia, $J_{x x}, J_{y y}$, and $J_{z z}$ are equal to $0.03699,0.03701$, and $0.00599 \mathrm{~kg} \cdot \mathrm{m}^{2}$, respectively. The output of the three body-mounted rate gyroscope is corrupted by a zero-mean Gaussian white noise with standard deviation $\sigma_{\epsilon} / \sqrt{\Delta t}=2.3 \cdot 10^{-6} \mathrm{rad} / \mathrm{s}$ where $\Delta t=0.25 \mathrm{~s}$ denotes the time increment between two successive gyro readouts. The Earth magnetic field mea- 


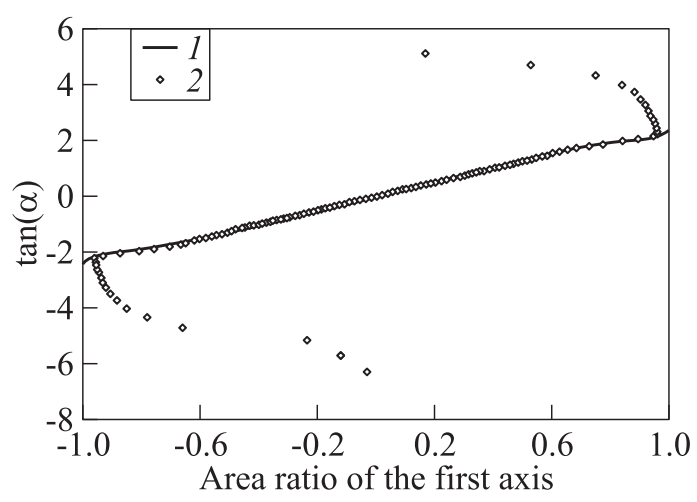

Figure 3 Comparison of the predicted (1) and measured (2) ratio $R_{2}$ as a function of the angle of incidence $\alpha$

surements, as read from a triad of magnetometers (MGM), are corrupted by zero-mean Gaussian white noises with an equivalent angular standard deviation $\sigma_{b} / \sqrt{\Delta t_{\mathrm{MGM}}}=1^{\circ}$ which is typical for coarse magnetic field measurements. The MGM sampling time, $\Delta t_{\mathrm{MGM}}$, was selected to be 20 times longer than the gyros' in order to test the filtering algorithm in computationally unfavorable conditions. The simulated Earth magnetic field corresponds to the IGRF11 model. The inertial coordinates frame corresponds to the local North-East-Nadir frame existing at the initial time, which is assumed to be the local ascending node time epoch. The spacecraft Sun vector measurement is acquired in parallel to the MGM measurements according to the study presented in the previous subsection. The simulations' runs last $12,000 \mathrm{~s}$ which corresponds to approximately two orbital revolutions of the satellite around the Earth. During that laps of time, the inertial projection of the spacecraft Sun vector is assumed constant.

The first simulated case assumes that the $\mathrm{S} / \mathrm{C}$ experiences free rotational dynamics around its center of mass with initial rates of $10 \mathrm{deg} / \mathrm{s}$ in all three axes. This corresponds to the maximum allowed rate magnitude after separation from the launcher. The second simulated case consists of a pure-spin scenario where the $\mathrm{S} / \mathrm{C}$ is spinning at the rate of $\sin (2 \pi / 150) \mathrm{deg} / \mathrm{s}$ around its axis of lowest inertia, which coincides with an arbitrary inertial direction. The sampling time of the MGM was reduced to $2.5 \mathrm{~s}$. The sampling time of the Sun sensors remained identical. For the sake of comparison, an additive extended Kalman filter (EKF) [4] was implemented, using the same conditions and statistical noise assumptions. Figure 4 shows the single run performances of three filters, the full quaternion filter (Full), the 2D-reduced quaternion filter using approach \#1 (RMAX), and the EKF. The error quaternion is computed via quaternion multiplication be- 

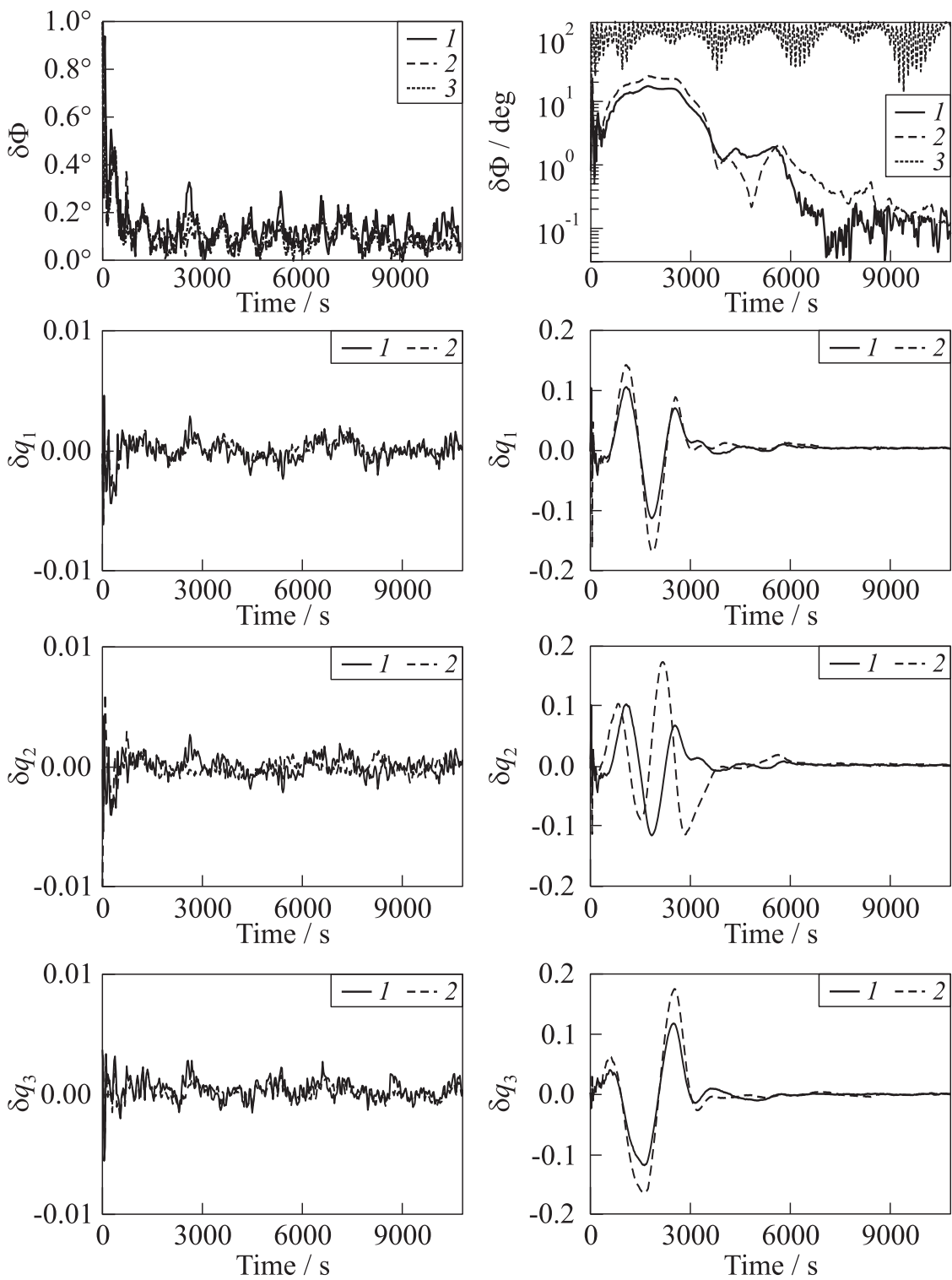

(a)

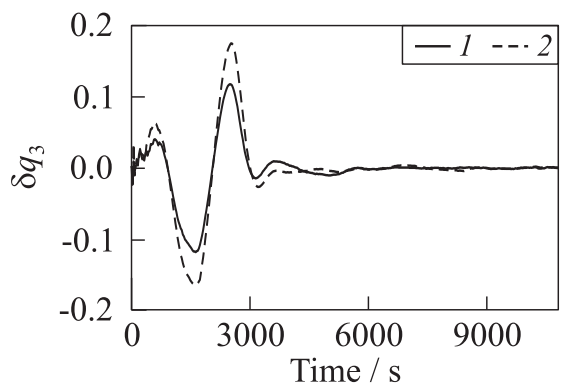

(b)

Figure 4 Time histories of the estimation quaternion-error on a single run in the spinning $(a)$ and tumbling (b) S/C: 1 - full filter; 2 - RMAX; and 3 - EKF. For the spinning $\mathrm{S} / \mathrm{C}$ case, the three filters show equivalent performances. For the freely tumbling S/C case, the RMAX filter shows similar performances to the full filter, and the EKF fails to converge 
tween the true and the normalized estimated quaternion. Its fourth component yields the angular estimation error, $\delta \Phi$, and the first three components form the vector part, $\delta \mathbf{q}(1), \delta \mathbf{q}(2)$, and $\delta \mathbf{q}(3)$. The three filters show similar performances in the spinning $\mathrm{S} / \mathrm{C}$ case. The angular error converges to a steady-state level of $0.1^{\circ}$ that is one tenth of the noise level present in the Earth magnetic field measurements and one third from the Sun sensor standard deviation. On the other hand, in the tumbling S/C case, the EKF fails to converge. This likely stems from its known sensitivity to initial conditions and modeling errors. The plots of the full and the RMAX filters show a slight advantage to the full filter. This result is supported by the analysis of the filters, previously described. The information is greater in the full filter than in the RMAX filter, which leads to a quicker convergence of the errors' first and second moments in the full filter. In steady-state, both filters reach similar levels of accuracy to those of the spinning $\mathrm{S} / \mathrm{C}$ case. A trade thus appears in the design of the quaternion filter where speed of convergence can be traded against computational burden (induced by the $4 D$-measurement filter). Notice that another nice feature of the RMAX filter is a better numerically conditioned algorithm since $H_{i j}(t)$ is a full row-rank matrix.

Figure $5 a$ depicts the time histories of $\sqrt{\operatorname{tr} P}$, i. e., the square-root of the trace of the a posteriori estimation error covariance matrix, for the various filters. All filters started with the same initial conditions for $P$ as well as for $X$, i. e, $P(0)=X(0)=5 I_{7}$. The curves 1 and 7 correspond to the full filter and to the RMAX filter, respectively. Each one of the other lines correspond to one of the possible 2D-measurement quaternion filters. For instance, the R1 filter implements at each update the submatrix obtained by extracting the rows 1 and 4 of the $4 \mathrm{D}$ observation matrix (see Table 1). The relative efficiency of the RMAX filter, within the family of the 2D-measurement filters, clearly appears in Fig. $5 a$. It is interesting to notice that the RMAX filter does not need to compute a specific measurement matrix, but merely picks the appropriate one from a limited number of possible values, as a result of the information maximization, and implements the appropriate update stage. By doing so, it differentiates itself at once from the other $2 \mathrm{D}$ filters, and the gap keeps increasing whenever each of the possible models shows a better observability. As can be seen from Fig. $5 a$, the full filter (1) converges quicker than any of the 2D-measurement filters, including the RMAX filter, and remains significantly lower asymptotically. This is not surprising since the information rate of that filter beneficiates from a richer (4D) observation matrix, i. e., its Frobenius norm is approximately twice that of any of its six $2 \times 4$ submatrices. This, therefore, yields a steeper convergence rate. In order to investigate the statistical consistency of the proposed maximal information filter, RMAX, the scenario of the tumbling S/C was simulated. The sampling rates of both the gyro and the MGM were chosen to be $10 \mathrm{~Hz}$. The sampling rate of the Sun sensors remained identical. These sampling rates are realistic and can even be further increased for typical gyro readouts. A set of 




(a)
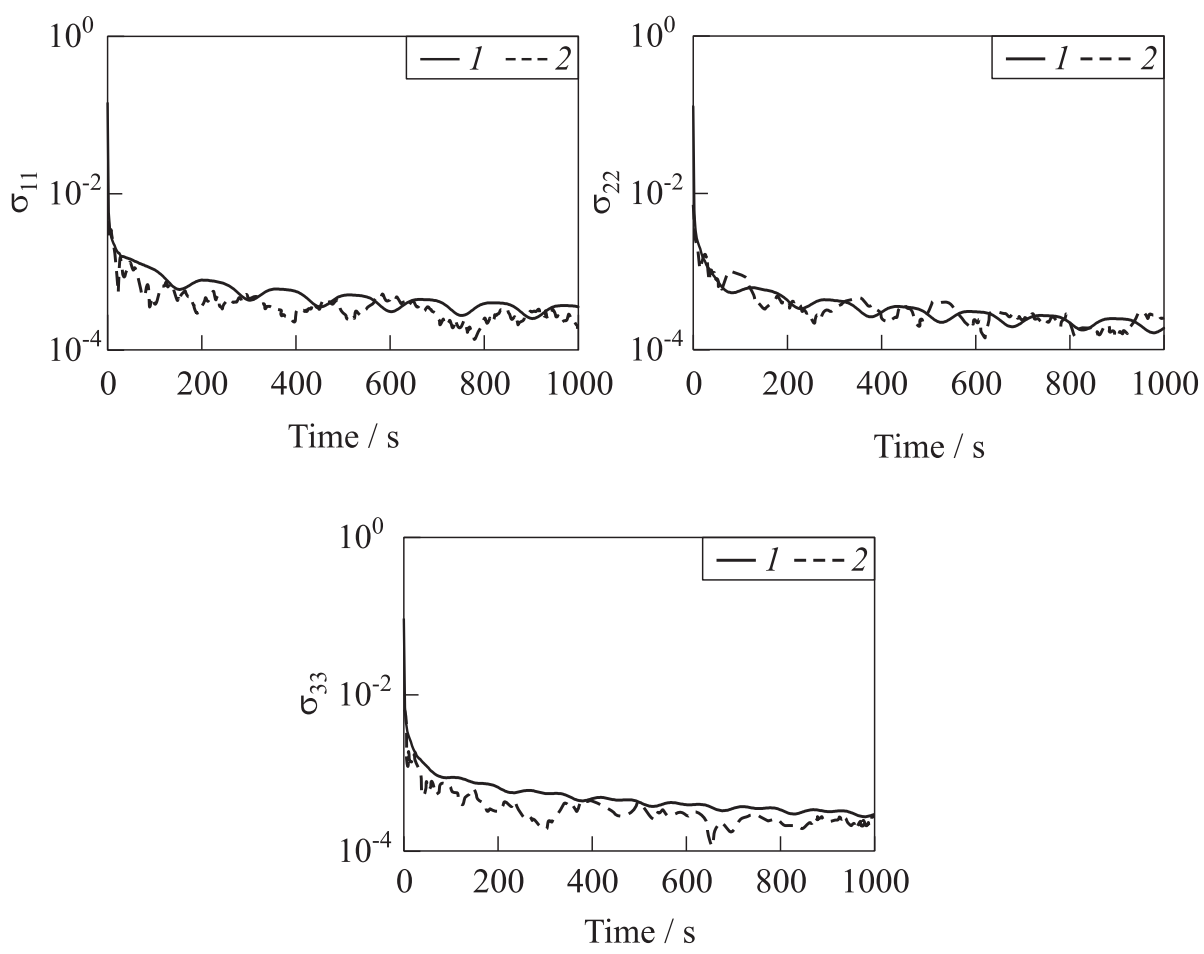

(b)

Figure 5 Second-order statistics. Single run comparison $(a)(1-$ full; $2-\mathrm{R} 1$; $3-\mathrm{R} 2 ; 4-\mathrm{R} 3 ; 5-\mathrm{R} 4 ; 6-\mathrm{R} 5$; and $7-\mathrm{RMAX})$ and Monte-Carlo consistency check $(b)(1$-filter; and 2 - Monte Carlo) 
60 Monte-Carlo simulations was run, with time-spans limited to $1000 \mathrm{~s}$ since convergence was reached far before.

Figure $5 b$ features the plots of the Monte-Carlo standard deviations of the quaternion-error vector part (curve 2) and of the corresponding (square-root) element in the $P_{t / t}$ matrix (curve 3 ). The two plots are close to each other which illustrates the statistical consistency of the proposed maximal information filter.

\section{CONCLUDING REMARKS}

This work accounts for current developments of the attitude determination system of the nanosatellite Delfi-n3Xt at TU Delft — Delft Institute of Technology. Novel results on quaternion Kalman filtering were presented. It is shown how to exactly carry through the covariance propagation in spite of the state dependence of the process noise. A nice property of this modeling is that the quaternion unit-norm property is preserved in the mean-square sense. Simple expressions were provided for the steady-state, where the second-order moment of the quaternion converges to a scalar matrix. This can be interpreted as the uncertainty being spread out equally among the quaternion components as time increases. In addition, the spectral properties of the $4 \mathrm{D} H$-matrix, as computed from a single vector observation, was exploited in order to devise several measurement order reduction techniques. Building on these novel results, a family of quaternion Kalman filters was developed, where the reduced measurement update stage significantly lowers the filter computational burden.

This paper also described part of the ongoing developments of a TU Delft designed Sun sensor system for the S/C Sun vector determination. At sensor level, which is developed following a four-quadrant design, the Sun line-of-sight is determined by a simple algorithm, where knowledge on uncertain physical parameters was alleviated. The proposed design takes into account geometrical imperfections due to manufacturing limitations. A thorough analysis of the errors associated with the photodiodes' measurement chain was presented, and approaches for Sun vector determination at S/C level were proposed. Numerical simulations, accounting for the Sun divergence but not the Earth albedo, showed a maximum angular error of approximately $0.3^{\circ}$ at 50 degree incidence. Experimental validation tests were also conducted showing satisfactory fit between measurement and model. Finally, a numerical example of attitude determination from vector observations - here, the Sun vector and the Earth magnetic field - was presented illustrating via extensive simulations the performances of the novel quaternion filters. The novel filter paradigm shows a clear advantage with respect to a standard additive Kalman filter, which failed to converge in a 
rapidly tumbling dynamics environment. Future work will handle a convergence analysis of the filtering algorithms, including the modeling of Earth albedo, and experimental investigations of the Sun vector determination and attitude estimation performances.

\section{ACKNOWLEDGMENTS}

This research was supported by the TU Delft Delfi Satellite Program and by The Israel Science Foundation (Grant No. 1546/08).

\section{REFERENCES}

1. Wertz, J. R., ed. 1984. Spacecraft attitude determination and control. Dordrecht, The Netherlands: D. Reidel.

2. Crassidis, J., F. L. Markley, and Y. Cheng. 2007. Nonlinear attitude filtering methods. J. Guidance Control Dyn. 30(1):12-28.

3. Lefferts, E. J., F. L. Markley, and M. D. Shuster. 1982. Kalman filtering for spacecraft attitude estimation. J. Guidance Control Dyn. 5:417-29.

4. Bar-Itzhack, I. Y., and Y. Oshman. 1985. Attitude determination from vector observations: Quaternion estimation. IEEE Trans. Aerospace Electronic Syst. 21:128-36.

5. Choukroun, D. 2008. Novel quaternion stochastic modelling and filtering. AIAA Paper No. 2008-6299.

6. Choukroun, D., Y. Oshman, and I. Y. Bar-Itzhack. 2006. Novel quaternion Kalman filter. IEEE Trans. Aerospace Electronic Syst. (1):174-90.

7. Mendel, J. M. 1987. Lessons in estimation theory for signal processing, communication, and Control. Englewood Cliffs, New Jersey: Prentice-Hall PTR.

8. Choukroun, D. 2009. Novel stochastic modeling and filtering of the attitude quaternion. J. Astronautical Sci. 57(1-2):167-89. 